\title{
Prognostic role and treatment tips of FHL1 expression in cytogenetically intermediate- and poor-risk acute myeloid leukemia
}

chengliang yin

1National Engineering Laboratory for Medical Big Data Application Technology, Chinese PLA General Hospital, Beijing,100853, China. 2Medical Big Data Center, Chinese PLA General Hospital, Beijing,100853, China.

Yuchen Liu

3Department of Hematology, Chinese PLA General Hospital, Beijing,100853, China.

\section{Qi Chen}

1National Engineering Laboratory for Medical Big Data Application Technology, Chinese PLA General Hospital, Beijing,100853, China. 2Medical Big Data Center, Chinese PLA General Hospital, Beijing,100853, China.

\section{Min Zhao}

1National Engineering Laboratory for Medical Big Data Application Technology, Chinese PLA General Hospital, Beijing,100853, China. 2Medical Big Data Center, Chinese PLA General Hospital, Beijing,100853, China.

\section{Jing Wu}

1National Engineering Laboratory for Medical Big Data Application Technology, Chinese PLA General Hospital, Beijing,100853, China. 2Medical Big Data Center, Chinese PLA General Hospital, Beijing,100853, China.

\section{Jinxiu Sun}

1National Engineering Laboratory for Medical Big Data Application Technology, Chinese PLA General Hospital, Beijing,100853, China. 2Medical Big Data Center, Chinese PLA General Hospital, Beijing,100853, China.

\section{Wanguo Xue}

1National Engineering Laboratory for Medical Big Data Application Technology, Chinese PLA General Hospital, Beijing,100853, China. 2Medical Big Data Center, Chinese PLA General Hospital, Beijing,100853, China.

\section{Liping Dou}

3Department of Hematology, Chinese PLA General Hospital, Beijing,100853, China.li

\section{Jinlong Shi ( $\square$ shijinlong@301hospital.com.cn )}

1National Engineering Laboratory for Medical Big Data Application Technology, Chinese PLA General Hospital, Beijing,100853, China. 2Medical Big Data Center, Chinese PLA General Hospital, Beijing,100853, 
China. 4Department of Biomedical Engineering, Chinese

\section{Research}

Keywords: Acute myeloid leukemia, drug resistance, prognostic biomarker, FHL1

Posted Date: March 13th, 2020

DOl: https://doi.org/10.21203/rs.3.rs-17167/v1

License: (c) (i) This work is licensed under a Creative Commons Attribution 4.0 International License. Read Full License 


\section{Abstract}

Background: Acute myeloid leukemia (AML) is a heterogeneous disorder of hematopoietic system, 35 to $40 \%$ of younger patients ( $<60$ years) are recurrent, refractory or drug-resistant, which are canonically called as cytogenetically intermediate- and poor-risk AML (IP-AML). Identifying novel biomarkers is an urgent clinical need and research hotspot for treatment optimization.

Methods: Bioinformatics analysis were carried out for RNA-Seq data derived from drug resistanceassociated cell lines and The Cancer Genome Atlas (TCGA). Varieties of comparison, visualization and functional enrichments were performed to Four-and-a-half LIM domain 1 (FHL1) and related genes. FHL1's expression changes were confirmed by 'wet' experiments. Kaplan-Meier method, log-rank test and multivariate Cox proportional hazards models were utilized to evaluate the associations between FHL1 expression and Overall Survival (OS), Event Free Survival (EFS), Relapse Free Survival (RFS). Prognostic significance of FHL1 expression was further validated in another independent larger IP-AML cohort (GSE6891). Moreover, construction of nomogram and validation of prognostic model.

Results: High expression of FHL1 (FHL1 high ) was a potentially effective biomarker of poor prognosis for IP-AML. Compared to FHL1 low group, FHL1 high was associated with short OS and EFS (145 patients, OS, $\mathrm{P}<0.001$; EFS, $\mathrm{P}<0.001$ ), which was further validated in GSE6891 (284 patients, $\mathrm{OS}, \mathrm{P}<0.001$; EFS, $\mathrm{P}<0.001)$. Multivariate analysis also confirmed the adverse prognosis of $\mathrm{FHL} 1$ high $(H R=2.2339, P=0.000156)$. ROC indicated an ideal predictive accuracy of the outcome model (AUC was 0.773). In addition, to understand the inherent mechanisms FHL1 involved, genome-wide characteristics were investigated to find that FHL1 might be involved in several important carcinogenic signal pathways.

Conclusions: FHL1's expression may serve as an effective prognostic biomarker, and could provide valuable treatment indications for IP-AML patients, whose distinctive gene patterns were helpful to further understand the recurrent, refractory and drug-resistant mechanisms for IP-AML.

\section{Background}

AML is a heterogeneous malignancy characterized by clonal expansion, infiltration of the bone marrow, blood and differentiation arrest of myeloid progenitors at various stages of incomplete maturation [1]. A major cause of treatment failure in patients with AML is the occurrence of multidrug resistance, which can can arise during chemotherapy or at relapse [2]. Recurrent and refractory disease status is an obstacle [1]. Especially, dysregulation of transcription mechanisms related to the pattern of mutation acquisition can affect apoptosis susceptibility and lead to drug resistance [3].

Drug resistance mechanism is unique to individuals drugs, different patients, and clinical risk factors such as white blood cell count, age, and other factors, which have been reported to affect drug resistance singly or in combination [4]. Especially, the treatment choice based on risk stratification, and targeted therapy may become less toxic and more effective when well defined genes are available [4]. Studies have already demonstrated several crucial molecule factors associated with favorable or adverse 
outcomes, which includes a variety of cytogenetic changes, such as mutations of TP53 [5], and/or ASXL1 [6], double CEBPA mutation [7], FLT3-ITD [8] and high expression of RUNX1 [9], as well as low expression of NPM1 [10]. These biomarkers can be useful indicators for risk stratification, but not cover all the AML patients. What's more, some genes that commonly involved in malignancy genetic regulation can not only make cells multilineage differentiation, but also help them to survive chemotherapy and expand during remission. These processes could eventually facilitate reducing relapse and curing the disease. Therefore, it is necessary in order to more thoroughly understand the underlying mechanism and to identify more target genes as potential biomarkers for the individualized IP-AML diagnosis and treatment.

FHL1 belongs to the FHL protein family, which are related to carcinogenesis and composed of four members, FHL1, FHL2, FHL3, and FHL5 in humans [11, 12]. Though some studies found FHL1 played important roles in embryonic development [13], suppression of cell differentiation [14] and oncogenesis [15], But, up to now, the role of FHL1 in clinical prognosis, risk stratification of IP-AML remains unclear [16].

This study confirmed the prognostic value of FHL1 expression in IP-AML patients, further explored the distinctive genomic patterns associated with FHL1 expression and explored the potential mechanisms resolving FHL1 via combining 'wet' experiment and bioinformatics analysis in IP-AML patients. Results provide direct evidence for using FHL1 expression as a new prognostic biomarker in risk stratification or a potential target for the precision diagnosis and treatment of IP-AML.

\section{Methods}

\section{Cell lines and cell culture}

The human leukemia cell line K562, and its anthracycline-resistant counterpart K562/A02 were obtained from Tianjin Institute of Hematology. Cells were all cultured in RPMI-1640 medium supplemented with $10 \%$ fetal calf serum (Gibco BRL, Grand Island, USA) in a humidified atmosphere containing $5 \% \mathrm{CO}_{2}$ at 37

${ }^{\circ} \mathrm{C}$. To maintain the multidrug resistance phenotype, doxorubicin was added to the culture media for $\mathrm{K} 562 / \mathrm{A} 02$ cells at the final concentration of $0.5 \mu \mathrm{g} / \mathrm{mL}$. The cells were cultured for 2 weeks in drug-free medium prior to their use in the experiments.

\section{Patients and treatment}

The first cohort was derived from TCGA (https://tcga-data.nci.nih.gov/tcga/) which was used as the training set, including 145 IP-AML in the 179 clinically annotated adult de novo AML patients. The validation data set included 284 IP-AML patients derived GSE6891

(https://www.ncbi.nlm.nih.gov/geo/query/acc.cgi?acc=gse6891), inlucding all the clinical, cytogenetic and molecular information. All these data were publicly accessible from the TCGA and GEO website.

Some molecule markers were available about the genetic risk of $\mathrm{FHL} 1^{\text {high }}$ and $\mathrm{FHL} 1^{\text {low }}$ groups, which was referred to NCCN(National Comprehensive Cancer Network)Acute Myeloid Leukemia Risk Status Guidelines Version 1.2018. 


\section{RNA analyses and gene expression by quantitative real-time reverse transcription polymerase chain reaction}

Total RNA from 3 K562/AO2 cell lines and 3 K562 cell lines was extracted with TRIZOL reagent (Invitrogen, Carlsbad, USA) and cDNA was synthesized by PrimeScript ${ }^{\text {TM }}$ RT reagent Kit (Takara) according to the manufacturer's instructions. The quantitative real-time polymerase chain reactions were then performed using KAPA SYBR FAST q-PCR Master Mix (2x) Kit. The following primers were used for quantitative PCR: FHL1, forward primer: 5' -TGCTGCCTGAAATGCTTTGAC - 3' and reverse primer: 5'GCCAGAAGCGGTTCTTATAGTG $-3^{\prime}$. We used the $2^{-\triangle C t}$ formula to examine the relative quantification of the target genes.

\section{Definition of clinical end points}

Descriptive statistics including frequency counts, median and range were appointed to describe patient characteristics, which were publicly accessible from the TCGA website. The main objective of this study was to explore the prognostic value of FHL1 expression in AML patients.

OS was defined as the time from date of diagnosis to death due to any cause or the last follow-up. EFS was the time from date of diagnosis to removal from the study because of the absence of complete remission, relapse or death. RFS was defined as the time from the date of diagnosis to removal from the study due to relapse.

To determine the best classification method, we subdivided AML patients into $\mathrm{FHL} 1^{\text {high }}$ and $\mathrm{FHL} 1^{\text {low }}$ groups according to the median based on FHL1 expression values. Patients with higher than median FHL1 expression values were classified as FHL $1^{\text {high }}$ group, and those with lower were classified as FHL $1^{\text {low }}$ group. High FLT3, NPM1, CEBPA, MLL-PTD, IDH1, IDH2, RUNX1, DNMT3A, TP53, ERG, BAALC, MN1, WT1, FLT3 expression levels were also determined based on the data. In the 179-patient TCGA cohort, $F H L 1^{\text {high }}$ patients were more frequent in AML FAB subtype M0 and less fell into FAB subtype M3 $(P=0.0001438, P=0.002776$, respectively). See supplementary Table $S 1$. In view of the therapeutic effect of FAB-M3 patient was very effective, this subtype datasets (16-AML cohort) was also deleted. In cytogenetically risk, good risk AML patients were deleted, and IP-AML patients (145-AML cohort) were finally just considered.

\section{Estimation of outcome signature for patients' prognosis, construction and assessment of the nomogram}

To investigate the associations between FHL1 expression levels and clinical, other molecular characteristics, the Fisher exact test was available for categories of variables and the Wilcoxon rank-sum test was available for continuous variables, respectively. The Kaplan-Meier method and log-rank test were utilized to estimate the association between FHL1 expression and OS, EFS. The proportional hazards assumption was verified for each variable before fitting Cox models. Multivariate Cox proportional hazards models were utilized to study the association between FHL1 expression levels and OS, EFS and 
RFS in the presence of other known risk factors. The forest was used to show the $\mathrm{P}$ value, $\mathrm{HR}$ and $95 \% \mathrm{Cl}$ of each variable through 'forestplot' $\mathrm{R}$ package. We used $\mathrm{R}$ software to perform the nomogram and evaluate the performance of the 4-year, 5-year and 6-year OS nomogram. Then receiver operating characteristic curve (ROC) analysis was used to calculate the area under curve (AUC) and check the prediction accuracy for our model.

\section{Bioinformatics analysis and statistical analyses}

Between FHL $1^{\text {high }}$ and FHL ${ }^{\text {low }}$ groups, the statistical cutoff values were a fold-change of 2 and an adjusted P-value of $<0.05$ (training data set). Hierarchical clustering based on expression levels of these mRNAs were performed and visualized by heatmap. Pathways were developed by Metascape, DAVID and Kyoto Encyclopedia of Genes and Genomes (KEGG). All analyses were performed using the R 3.4.4 software packages.

\section{Results}

\section{Expression of FHL1 in K562 and K562/A02 AML cell lines}

RNA-Seq was carried out and compared in the drug resistance cell lines (K562/A02) and the non-resistant cell lines (K562) (each has 3 biological replicates) (Fig. 1). Up-regulation genes (drug resistanceassociated genes) were filtered out (Fig. 1a). Remarkably higher mRNA expression of FHL1 was evident in K562/A02 cell lines than K562 cell lines $(P=0.03)$ (Fig. 1b). Real-time quantitative PCR further confirmed that FHL1 was expressed at high mRNA expression levels in drug resistant group $(P=0.02427$, 3 K562/A02 VS 3 K562) (Fig. 1C). All these results showed significant mRNA overexpression of FHL1. RNA study of 2 human leukemia cell lines found SPARC and FHL1 were two eminent rank up-regulated genes (Fig. 1a). However, SPARC has previously been in-depth studied in acute myeloid leukemia, so we decide to study gene FHL1 [17].

\section{Differences of clinical and molecular characteristics between FHL1 ${ }^{\text {high }}$ and FHL $1^{\text {low }}$ groups}

Clinical characteristics of 145 IP-AML patients in TCGA dataset with FHL $1^{\text {high }}$ were summarized in Table 1. No significant differences were noted in clinical information including age, WBC count, BM blasts and PB blasts between the two groups. FHL ${ }^{\text {high }}$ patients were more common in AML FAB MO $(P=$ 0.0001212). FHL ${ }^{\text {high }}$ patients seemed likely to have a lower prevalence of FLT3-ITD, NPM1 and biallelic CEBPA mutation than FHL ${ }^{\text {low }}$ patients $(P<0.05)$. (See Table 1)

With regard to cytogenetic characteristics, $\mathrm{FHL} 1^{\text {high }}$ patients had a tendency for higher frequency of complex cytogenetics $(P=0.01282)$, and seemed more likely to have a higher prevalence mutation of RUNX1, and accompanied with higher expression levels of BGR, BAALC and MN1 than FHL1 ${ }^{\text {low }}$ patients $(P<0.05)$. (See Table 1) 
Table 1

Clinical and molecular characteristics in intermediate- and poor-risk TCGA-AML cohort according to FHL1 expression levels

\begin{tabular}{|c|c|c|c|}
\hline Variable & $\mathrm{FHL}_{1}{ }^{\mathrm{Low}}(\mathrm{n}=73)$ & $\mathrm{FHL} 1^{\mathrm{High}}(\mathrm{n}=72)$ & $\mathbf{P}$ \\
\hline Age, median y (range) & $55(21-82)$ & $57.8(18-88)$ & 0.298 \\
\hline WBC count, median $X 10^{9} / \mathrm{L}$ (range) & $45.3(1.2-297.4)$ & $33.6(0.6-171.9)$ & 0.529 \\
\hline BM blasts, median \%( range) & $72(30-100)$ & $73(32-97)$ & 0.766 \\
\hline PB blasts, median \%( range) & $40(0-98)$ & $39(0-94)$ & 0.280 \\
\hline \multicolumn{4}{|l|}{ FAB subtype, no (\%) } \\
\hline M0 & 1 & 15 & 0.0001212 \\
\hline M1 & 21 & 19 & 0.853 \\
\hline M2 & 20 & 14 & 0.3276 \\
\hline M4 & 15 & 12 & 0.6704 \\
\hline M5 & 14 & 7 & 0.1559 \\
\hline M6 & 1 & 1 & 1.000 \\
\hline M7 & 1 & 2 & 1.000 \\
\hline \multicolumn{4}{|l|}{ Fusion gene, no (\%) } \\
\hline BCR-ABL1 & 1 & 2 & 1.000 \\
\hline \multicolumn{4}{|l|}{ Mutated gene, no (\%) } \\
\hline FLT3-ITD & 24 & 5 & 0.0001231 \\
\hline FLT3-TKD & 22 & 19 & 0.7129 \\
\hline NPM1 & 34 & 14 & 0.0007282 \\
\hline Biallelic CEBPA & 11 & 2 & 0.01699 \\
\hline IDH1 & 10 & 6 & 0.4276 \\
\hline IDH2 & 8 & 9 & 0.802 \\
\hline RUNX1 & 0 & 15 & $1.214 \mathrm{e}-05$ \\
\hline DNMT3A & 25 & 18 & 0.2759 \\
\hline
\end{tabular}

FAB, French-American-British classification; ITD, internal tandem duplication; WT: wild type. High FLT3, NPM1, CEBPA, MLL-PTD, IDH1, IDH2, RUNX1, DNMT3A, TP53, ERG, BAALC, MN1, WT1, FLT3 expression were defined as an expression level above the median of all samples 


\begin{tabular}{|c|c|c|c|}
\hline Variable & FHL1 $^{\text {Low }}(n=73)$ & $\mathrm{FHL} 1^{\mathrm{High}}(\mathrm{n}=72)$ & $\mathbf{P}$ \\
\hline TP53 & 4 & 11 & 0.06094 \\
\hline KIT & 1 & 1 & 1 \\
\hline ASXL1 & 0 & 3 & 0.1198 \\
\hline TET2 & 10 & 5 & 0.2754 \\
\hline High ERG, no (\%) & 26 & 44 & 0.002752 \\
\hline High BAALC, no (\%) & 21 & 49 & $2.682 \mathrm{e}-06$ \\
\hline High MN1, no (\%) & 22 & 48 & $1.359 e-05$ \\
\hline High WT1, no (\%) & 34 & 36 & 0.7407 \\
\hline Normal karyotype, no (\%) & 49 & 30 & 0.002668 \\
\hline Complex karyotype, no (\%) & 6 & 17 & 0.01282 \\
\hline \multicolumn{4}{|c|}{ Risk status based on cytogenetic and molecular abnormalities, no (\%) } \\
\hline Intermediate-risk & 55 & 45 & 0.7366 \\
\hline Poor-risk & 17 & 28 & 0.04943 \\
\hline \multicolumn{4}{|c|}{$\begin{array}{l}\text { FAB, French-American-British classification; ITD, internal tandem duplication; WT: wild type. High } \\
\text { FLT3, NPM1, CEBPA, MLL-PTD, IDH1, IDH2, RUNX1, DNMT3A, TP53, ERG, BAALC, MN1, WT1, FLT3 } \\
\text { expression were defined as an expression level above the median of all samples }\end{array}$} \\
\hline
\end{tabular}

\section{High expression of FHL1 was associated with poor prognosis in IP-AML patients}

Survival analyses were performed in the cohort of $145 \mathrm{IP}-\mathrm{AML}$. FHL ${ }^{\text {high }}$ patients group displayed remarkable shorter OS (Fig. 2a, $\mathrm{P}<0.001$ ) and EFS (Fig. $2 \mathrm{~b}, \mathrm{P}<0.001$ ) than the $\mathrm{FHL} 1^{\text {low }}$ patients group. Compared to the $\mathrm{FHL} 1^{\text {low }}$ groups, the $\mathrm{FHL} 1^{\text {high }}$ patient group had more RFS (Fig. $2 \mathrm{c}, \mathrm{P}<0.05$ ). In addition, the increase was linked to worse OS of patients in the Kaplan-Meier survival analysis after controlling for multiple hypothesis tests (Fig. 2d). FHL1 expression and age, WBC, FLT3, TP53, CEBPA mutations were significantly different in Hazard ratio (Fig. 2d, $P<0.01$ ). A larger cohort of 284 AML samples (GSE6891, validation data set) was further studied, results also showed that $\mathrm{FHL} 1^{\text {high }}$ was significantly associated with shorter OS and EFS (Fig. 3, P<0.01).

\section{Multivariable analysis confirmed the prognostic value of FHL1 ${ }^{\text {high }}$}

Adjusting for known prognosticators by multivariable analyses, including commonly known mutations and several recently published prognostic indicators such as mutations of NPM1, FLT3, CEBPA, TP53, 
FHL1 expression were still independent prognostic factors.

In the multivariate model for OS, FHL ${ }^{\text {high }}$ IP-AML patients had 2.2339 times increase of risk to death $(\mathrm{P}<$ 0.001 , Table 2). Other hazards correlated with OS including older patients and WBC, TP53 mutations ( $P=$ $0.009267, P=0.000618$, respectively, Table 2$)$ and CEBPA biallelic mutations $(P=0.000156$, Table 2$)$. In the multiple models of EFS, high expression of FHL1 remained a dramatically worse prognosticator $(\mathrm{P}<$ 0.001 , Table 2) after the adjustment of other risk factors. Similarly, the presence of older patients, TP53 and CEBPA biallelic mutations were associated with poor $E F S(P=0.00265, P=0.0000416$, respectively, Table 2).

Moreover, we also conducted an analysis, including IP-AML patients whose survival was $>30$ days in order to ignore the cause of induction death by intense chemotherapy. As a result, high expression of FHL1 was still independently associated with adverse impacts on OS [HR $(95 \% \mathrm{Cl}), 1.0152(1.0114$, 1.044), $P=0.025629]$, EFS [HR $(95 \% \mathrm{Cl}), 1.0146(1.0017,1.028), P=0.026483]$.

Table 2

Multivariable analysis of OS and EFS in the cohort of 145 IP-AML patients

\begin{tabular}{|c|c|c|c|c|c|c|c|c|}
\hline $\begin{array}{l}\text { Variables in } \\
\text { Final Model by } \\
\text { End Point }\end{array}$ & HR & $95 \% \mathrm{Cl}$ & & $\mathbf{P}$ & HR & $95 \% \mathrm{Cl}$ & & $\mathbf{P}$ \\
\hline & os & & & & EFS & & & \\
\hline $\begin{array}{l}\text { FHL1 } \\
\text { expression } \\
\text { group }\end{array}$ & 2.2339 & 1.4728 & 3.388 & 0.000156 & 2.512 & 1.6171 & 3.902 & 0.0000416 \\
\hline Age, $\otimes 60 ; \geq 60$ & 1.0316 & 1.0167 & 1.047 & 2.78E-05 & 1.032 & 1.0168 & 1.047 & 0.0000295 \\
\hline WBC & 1.0062 & 1.0015 & 1.011 & 0.009267 & 1.007 & 1.0022 & 1.011 & 0.0035 \\
\hline $\begin{array}{l}\text { FLT3-ITD, } \\
\text { presented VS } \\
\text { others }\end{array}$ & 1.304 & 0.9904 & 1.717 & 0.05856 & 1.457 & 1.1106 & 1.912 & 0.00659 \\
\hline $\begin{array}{l}\text { TP53,mutated } \\
\text { VS wild type }\end{array}$ & 2.8813 & 1.5721 & 5.281 & 0.000618 & 2.484 & 1.3724 & 4.497 & 0.00265 \\
\hline $\begin{array}{l}\text { NPM1,mutated } \\
\text { VS wild type }\end{array}$ & 1.3292 & 0.5972 & 2.958 & 0.485661 & 1.535 & 0.6977 & 3.377 & 0.28682 \\
\hline $\begin{array}{l}\text { CEBPA,biallelic } \\
\text { mutated VS } \\
\text { others }\end{array}$ & 2.2339 & 1.4728 & 3.388 & 0.000156 & 2.512 & 1.6171 & 3.902 & 0.0000416 \\
\hline
\end{tabular}

\section{Construction of nomogram and validation of prognostic model}


According to the results of cox regression analysis and forest plot, we further construct a nomogram combining independent prognostic factors, including potential factor patient's age, WBC and gene NPM1, CEBPA, TP53, FLT3, FHL1 to provide a quantitative method for the clinicians to predict the probability of 4-year, 5-year and 6-year OS in IP-AML patients (Fig. 4a). Every patient would get a total point by plus each prognostic parameters point, and the higher total points mean a worse outcome for that patient. Moreover, ROC analysis showed that the prediction accuracy of the outcome model and AUC was 0.773 (Fig. 4b).

\section{Associations between genome-wide gene-expression profiles and FHL1 expression}

To further assess the biological role of FHL1 in leukemogenesis, we derived FHL1-associated geneexpression differential analysis based on the comparison of RNA expression between FHL $1^{\text {high }}$ and FHL $1^{\text {low }}$ patients group (training data set). A total of 172 up-regulated and 87 down-regulated genes were considered to be significantly associated with FHL1 expression (Fig. 5a). Further, these aberrant genes were presented as an expression heatmap (Fig. 5b). These up-regulated genes included genes known as leukemia-associated biomarkers, such as: (1) CCND1, which played a crucial role in the regulation of cell proliferation and hematopoietic differentiation, and CCND1-BCL2 gene network was a direct target of amifostine treating human acute megakaryocytic leukemia [18]; (2) MLLT3, which was a proto-oncogene, was first reported in leukemia and involved in many different cellular processes, such as cell differentiation [19];(3) AKT3, was a key regulator of signal transduction pathways. Its tight control over cell proliferation and cell viability is manifold [20]. However, some tumor suppressors were downregulated, such as: (1) DDIT3, which emerged as a regulatory node with positive linkage to erythroid regulators and negative association with myeloid determinants [21]; (2) CEBPA, a decrease of CEBPAdependent HK3 expression promoted primary AML.

Cell signal pathways, acting as functional units of gene groups, play important biological roles in major cellular processes, including cancer development and progression. Thus, we assessed the important cell signal pathways especially focusing on some associated with FHL1. The up-regulated genes involved in some significant pathways were as followings: (1) Platelet activation, signaling and aggregation (GNAl1); (2) JAK-STAT signaling pathway (CCND1, FHL1, IL2RA, IL6ST, AKT3); (3) VEGF signaling pathway (AKT3); (4) MAPK signaling pathway (GNA12, AKT3); (5) PI3K-Akt signaling pathway (CCND1, AKT3). The down-regulated genes including the following: transcriptional misregulation in cancer (CEBPA, DDIT3) (Fig. 5c-d).

In K562/A02 cell lines, we found the top rank of differences pathways PI3K-Akt signaling pathway and JAK-STAT signaling pathway (Fig. 5c). In KEGG, it was interesting that cell signaling pathways of FHL1 expression were involved in "PI3K-Akt signaling pathway" and "MAPK signaling pathway", both of which were included in "JAK-STAT signaling pathways" (CCND1, FHL1, IL2RA, IL6ST, AKT3) (Fig. 5d). These 
dysregulated genes and pathways were consistent with their known understanding about leukemogenesis, which might explain the involvement between FHL1 and the prognosis of IP-AML.

\section{Discussion}

The majority of AML patients still succumb to this disease because of refractoriness to therapy or relapse, and new therapeutic approaches for AML are an urgent clinical need. Our study provided direct evidences that $\mathrm{FHL} 1^{\text {high }}$ predicted adverse outcomes for AML. Firstly, FHL1 was widely expressed at high levels in drug resistant cell lines (Fig. 1b). Meanwhile, quantitative RT-PCR was confirmed consistently that FHL1 as an important gene was differed dramatically and expressed at high level in drug resistant group (Fig. 1C). All these supported that FHL1 was a vital drug resistance gene and significantly overexpressed.

Secondly, for better studying the correlation between FHL1 expression and the prognosis of IP-AML, including some pretreatment molecular characteristics, the prognostic value of FHL1 was further confirmed in the independent group. In these $145 \mathrm{IP}$-AML patients, we found that FHL $1^{\text {high }}$ was linked to the presence of other adverse prognosticators. With regard to molecular factors, FHL1 ${ }^{\text {high }}$ patients had a lower prevalence of FLT3-ITD, NPM1 and Biallelic CEBPA mutation and higher prevalence mutation of RUNX1, higher expression levels of BAALC and MN1 than FHL1 ${ }^{\text {low }}$ patients (Table 1). These findings indicated that FHL1 might play an active role in leukemogenesis just like other tumor markers in IP-AML patients.

Further, patients with FHL $1^{\text {high }}$ were significantly more classified in the FAB MO subgroups than with $\mathrm{FHL} 1^{\text {low }}$, suggesting that the leukemic FHL1 patients derived from relatively more minimally differentiated IP-AML patients (Table 1), which might indicate adverse malignancy. In addition, based on TCGA cohorts, FHL1 ${ }^{\text {high }}$ was proved to have significant associations with adverse outcomes. FHL $1^{\text {high }}$ was associated with shorter OS, EFS and RFS, noteworthy difference in OS and EFS (Fig. 2a- C, Fig. 3). This study demonstrated that FHL1 might play an oncogenic role in leading to adverse prognosis in the development of leukemia. Meanwhile, negative factors (Age, WBC and FLT3, TP53, CEBPA mutations) were associated with poor OS and EFS. After adjusting for known prognosticators by multivariable analyses, the association of FHL $1^{\text {high }}$ with adverse OS and EFS still existed and FHL $1^{\text {high }}$ expressers harbored poor OS and EFS. In addition, the nomogram based on the model exhibits an impressive performance and clinical applicability. Moreover, prognostic significance in FHL1 was further consistently validated in another larger 284 IP-AML cohort ((Fig. 3). All these results proved FHL1 as a potential prognosticator and therapeutic target of IP-AML, which could promote further fine stratification of NCCN cytogenetically IP-group.

Disease recurrence occurs in most IP-AML patients within 3 years after diagnosis. A short duration of remission (i.e., $<6$ months), adverse genetic factors, older age, and poor general health status are the major determinants of outcome after relapse [22]. Ignoring the cause of induction death by intense chemotherapy by AML patients whose survival was $>30$ days, we also found that high expression of 
FHL1 was still independently associated with short OS and EFS. Taken together, clinical characteristics of FHL1 ${ }^{\text {high }}$ expressers implied FHL1 expression level might be associated with a poor outcome and results supported highlight FHL1 functions acting as a potent oncogenic role in leukemia development. Above all, these also showed that FHL1 acted as an independent adverse prognostic biomarker, which might substitute other adverse factors and might be employed to improve risk stratification of NCCN IP-patients.

Some studies also found that FHL1 in mRNA was up-regulated during embryonic skeletal muscle development [23], human hypertrophic cardiomyopathy and cardiovascular system [24]. Meanwhile, FHL1 was noticeably down-regulated in a variety of cancers including lung [25], breast [26, 27], colon [28] and gastric cancers [29]. This paper supported that FHL1 was up-regulated and acted as a potent oncogenic role in leukemia development. Here, we further studied functional pathway of FHL1.

In cell lines, this paper found top rank of differences pathways (PI3K-Akt signaling pathway and JAKSTAT signaling pathway), which might be connected with drug resistance (Fig. 5c), and FHL1 was involved in JAK-STAT signaling pathway (CCND1, FHL1, IL2RA, IL6ST, AKT3) (Fig. 5d), which had shown promising prognostic values. In KEGG, surprise was that cell signaling pathways of FHL1 expression were involved in "PI3K-Akt signaling pathway" and "MAPK signaling pathway", both of which were included in "JAK-STAT signaling pathways" (Fig. 5d). Gene CCND1 played a vital role in the regulation of cell proliferation and hematopoietic differentiation [18, 30]. AKT3 control over cell proliferation and cell viability was manifold [20]. In the JAK-STAT pathway, following the binding of cytokines to their cognate receptor, STATs were enabled to modulate the expression of target genes by members of the JAK family of tyrosine kinases. In addition to the activation of STATs, JAKs mediate the recruitment of other molecules such as the MAP kinases, PI3 kinase etc (PI3K-Akt signaling pathway and MAPK signaling pathway existed in the JAK-STAT pathway). These molecules process downstream signals via the RasRaf-MAP kinase and PI3 kinase pathways, led to the activation of additional transcription factors. PI3KAkt-mTOR pathway amplification was associated with reduced OS and DFS [20]. So we speculated that PI3K-Akt signaling pathway and MAPK signaling pathway played a pivotal role in JAK-STAT signaling pathway. In platelet activation pathway and another cancer pathway (VEGF signaling pathway also was found, including PI3K-Akt signaling pathway and MAPK signaling pathway) (Fig. 4d), FHL1 played a driver role in the aggressiveness of cancer cells and a potential catalyzes role in tumor angiogenesis. In such a scenario, gene mutations would cooperate with the genes and protein expression profile to present a cellular phenotype that depended on both components. In the present study, FHL1 as a target gene of downstream of TLX1 also acted as anti-oncogene was a dual function regulator, serving as either an activator or a repressor depending on cell and promoter context [31]. Together with these genes and pathways, FHL1 was the key gene in signaling pathway, especially in PI3K-Akt signaling pathway and JAK-STAT signaling pathway and might play a crucial role in the cancer process. Gene knockout and pathological experiments may also be further studied to determine the function of FHL1. Our results proved FHL1 as a potential prognosticator and therapeutic target for IP-AML and provided new perspectives into the pathogenesis and precision medicine of IP-AML. 


\section{Conclusions}

In summary, our study provided evidences that high FHL1 expression was associated with drug resistance and carried various kinds of molecular adverse events and could predict unfavorable survival in IP-AML patients, even after adjusting for other known molecular risk factors. In future, FHL1 might be used to diagnose human clinical disease to evaluate molecular genetic lesions as a prognostic and predictive marker, as well as open an innovative avenue of investigations aiming at developing therapies that target cancer cells. We just hope that exciting developments in our understanding of the molecular pathogenesis of IP-AML will translate into clinical practice in the future. Taken together, FHL1 plays an important role in leukemogenesis and will be potentially utilized as a therapeutic target for IP-AML.

\section{Declarations}

\section{Ethics approval and consent to participate}

This study was approved by the institutional review boards of all participating institutions.

\section{Consent for publication}

All the authors in this paper consent to publication of the work.

\section{Availability of data and materials}

The datasets used and/or analysed during the current study are available from the corresponding author on reasonable request.

\section{Competing interests}

The authors declare that they have no competing interests.

\section{Funding}

This work was supported by the National Key Scientific Instrument and Equipment Development Project (6192780187) and National Key Research and Development Program of China (2017YFC0112905 and 2017YFC0908400).

\section{Authors' contributions}


JLS and LPD designed the study. CLY performed the study and analyzed the data. CLY and JLS wrote the manuscript. WGX and LPD provided the expert consultations and clinical suggestions. All authors reviewed the final version of the manuscript.

\section{Acknowledgements}

Not applicable.

\section{Author details}

${ }^{1}$ National Engineering Laboratory for Medical Big Data Application Technology, Chinese PLA General Hospital, Beijing, 100853, China. ${ }^{2}$ Medical Big Data Center, Chinese PLA General Hospital, Beijing, 100853 , China. ${ }^{3}$ Department of Hematology, Chinese PLA General Hospital, Beijing, 100853, China. ${ }^{4}$ Department of Biomedical Engineering, Chinese PLA General Hospital, Beijing,100853, China.

\section{References}

1. Magliulo D, Bernardi R, Messina S: Lysine-Specific Demethylase 1A as a Promising Target in Acute Myeloid Leukemia. Frontiers in Oncology 2018, 8:255.

2. van der Kolk DM, de Vries EG, Muller M, Vellenga $E$ : The role of drug efflux pumps in acute myeloid leukemia. Leuk Lymphoma 2002; 43(4): 685-701.

3. Look AT: Oncogenic Transcription Factors in the Human Acute Leukemias. Science 1997, 278:10591064.

4. Funato T, Takeda M: Approaches to detect the drug resistance in acute leukemia. Journal of Electrophoresis 2005, 49:85-93.

5. Montalban-Bravo G, Takahashi K, Garcia-Manero G: Decitabine in TP53-Mutated AML. N Engl J Med 2017, 376:796-797.

6. Thol F, Friesen I, Damm F, Yun H, Weissinger EM, Krauter J, Wagner K, Chaturvedi A, Sharma A, Wichmann M: Prognostic significance of ASXL1 mutations in patients with myelodysplastic syndromes. Journal of Clinical Oncology 2011, 29:2499-2506.

7. Barjesteh van Waalwijk van Doorn-Khosrovani S, Erpelinck C, Meijer J, van Oosterhoud S, van Putten WL, Valk PJ, Berna Beverloo H, Tenen DG, Lowenberg B, Delwel R: Biallelic mutations in the CEBPA gene and low CEBPA expression levels as prognostic markers in intermediate-risk AML. Hematol $\mathrm{J}$ 2003, 4:31-40.

8. Weisberg E, Sattler M, Ray A, Griffin JD: Drug resistance in mutant FLT3-positive AML. Oncogene 2010, 29:5120-5134. 
9. Huang G, Shigesada K, Ito K, Wee HJ, Yokomizo T, Ito Y: Dimerization with PEBP2 $\beta$ protects RUNX1/AML1 from ubiquitin-proteasome-mediated degradation. Embo Journal 2001, 20:723-733.

10. Verhaak RG, Goudswaard CS, Van PW, Bijl MA, Sanders MA, Hugens W, Uitterlinden AG, Erpelinck CA, Delwel R, Löwenberg B: Mutations in nucleophosmin (NPM1) in acute myeloid leukemia (AML): association with other gene abnormalities and previously established gene expression signatures and their favorable prognostic significance. Blood 2005, 106:3747-3754.

11. Kadrmas JL, Beckerle MC: The LIM domain: from the cytoskeleton to the nucleus. Nat Rev Mol Cell Biol 2004, 5:920-931.

12. Lin J, Ding L, Jin R, Zhang H, Cheng L, Qin X, Chai J, Ye Q: Four and a half LIM domains 1 (FHL1) and receptor interacting protein of $140 \mathrm{kDa}$ (RIP140) interact and cooperate in estrogen signaling. Int $J$ Biochem Cell Biol 2009, 41:1613-1618.

13. Brown S, ., Biben C, ., Ooms LM, Maimone M, ., Mcgrath MJ, Gurung R, ., Harvey RP, Mitchell CA: The cardiac expression of striated muscle LIM protein 1 (SLIM1) is restricted to the outflow tract of the developing heart. Journal of Molecular \& Cellular Cardiology 1999, 31:837-843.

14. Grossi M, Hiou-Feige A, Tommasi Di Vignano A, Calautti E, Ostano P, Lee S, Chiorino G, Dotto GP: Negative control of keratinocyte differentiation by Rho/CRIK signaling coupled with up-regulation of KyoT1/2 (FHL1) expression. Proc Natl Acad Sci U S A 2005, 102:11313-11318.

15. Morgan MJ, Whawell SA: The Structure of the Human LIM Protein ACT Gene and Its Expression in Tumor Cell Lines \. Biochemical \& Biophysical Research Communications 2000, 273:776-783.

16. Cowling BS, Mcgrath MJ, Nguyen MA, Cottle DL, Kee AJ, Brown S, Schessl J, Zou Y, Joya J, Bönnemann CG: Identification of FHL1 as a regulator of skeletal muscle mass: implications for human myopathy. Journal of Cell Biology 2008, 183:1033.

17. Alachkar H, Santhanam R, Maharry K, Metzeler KH, Marcucci G: SPARC promotes leukemic cell growth and predicts acute myeloid leukemia outcome. The Journal of clinical investigation 2014, 124:1512-1524.

18. Zhang F, Yang B, Zhang K, Hou ML, Lu XC, Li YX: CCND1-BCL2 Gene Network: a direct target of Amifostine in human acute megakaryocytic leukemia cells. Chemical Biology \& Drug Design 2017, 89:681-693.

19. Pina C, May G, Soneji S, Hong D, Enver T: MLLT3 Regulates Early Human Erythroid and Megakaryocytic Cell Fate. Cell Stem Cell 2008, 2:264-273.

20. Bertacchini J, Heidari N, Mediani L, Capitani S, Shahjahani M, Ahmadzadeh A, Saki N: Targeting PI3K/AKT/mTOR network for treatment of leukemia. Cellular \& Molecular Life Sciences Cm/s 2015, 72:2337-2347.

21. Pina C, Teles J, Fugazza C, May G, Wang D, Guo Y, Soneji S, Brown J, Edén P, Ohlsson M, et al: SingleCell Network Analysis Identifies DDIT3 as a Nodal Lineage Regulator in Hematopoiesis. Cell reports 2015, 11:1503-1510.

22. Döhner H, Estey EH, Amadori S, Appelbaum FR, Büchner T, Burnett AK, Dombret H, Fenaux P, Grimwade D, Larson RA, et al: Diagnosis and management of acute myeloid leukemia in adults: 
recommendations from an international expert panel, on behalf of the European LeukemiaNet. Blood 2010, 115:453-474.

23. Chu PH, Ruizlozano P, Zhou Q, Cai C, Chen J: Expression patterns of FHL/SLIM family members suggest important functional roles in skeletal muscle and cardiovascular system. Mechanisms of Development 2000, 95:259-265.

24. Lim DS, Roberts R, Marian AJ: Expression Profiling of Cardiac Genes in Human Hypertrophic Cardiomyopathy: Insight Into the Pathogenesis of Phenotypes. Acc Current Journal Review 2002, 11:48-49.

25. Niu C, Liang C, Guo J, Cheng L, Zhang H, Qin X, Zhang Q, Ding L, Yuan B, Xu X: Downregulation and growth inhibitory role of FHL1 in lung cancer. International Journal of Cancer 2012, 130:2549-2556.

26. Li X, Jia Z, Shen Y, Ichikawa H, Jarvik J, Nagele RG, Goldberg GS: Coordinate suppression of Sdpr and Fhl1 expression in tumors of the breast, kidney, and prostate. Cancer Science 2008, 99:13261333.

27. Ding L, Chang N, Zheng Y, Xiong Z, Liu Y, Lin J, Sun H, Huang K, Yang W, Li X: FHL1 interacts with oestrogen receptors and regulates breast cancer cell growth. Journal of Cellular \& Molecular Medicine 2011, 15:72-85.

28. Wilczek E, Rzepko R, Nowis D, Legat M, Golab J, Glab M, Gorlewicz A, Konopacki F, Mazurkiewicz M, Sladowski $\mathrm{D}$ : The possible role of factor $\mathrm{H}$ in colon cancer resistance to complement attack. International Journal of Cancer 2008, 122:2030-2037.

29. Xu Y, Zhe L, Guo K: Expression of FHL1 in gastric cancer tissue and its correlation with the invasion and metastasis of gastric cancer. Molecular \& Cellular Biochemistry 2012, 363:93-99.

30. Deshpande A, Sicinski P, Hinds PW: Cyclins and cdks in development and cancer: a perspective. Oncogene 2005, 24:2909.

31. Rice KL, Kees UR, Greene WK: Transcriptional regulation of FHL1 by TLX1/HOX11 is dosage, cell-type and promoter context-dependent. Biochem Biophys Res Commun 2008, 367:707-713.

\section{Figures}



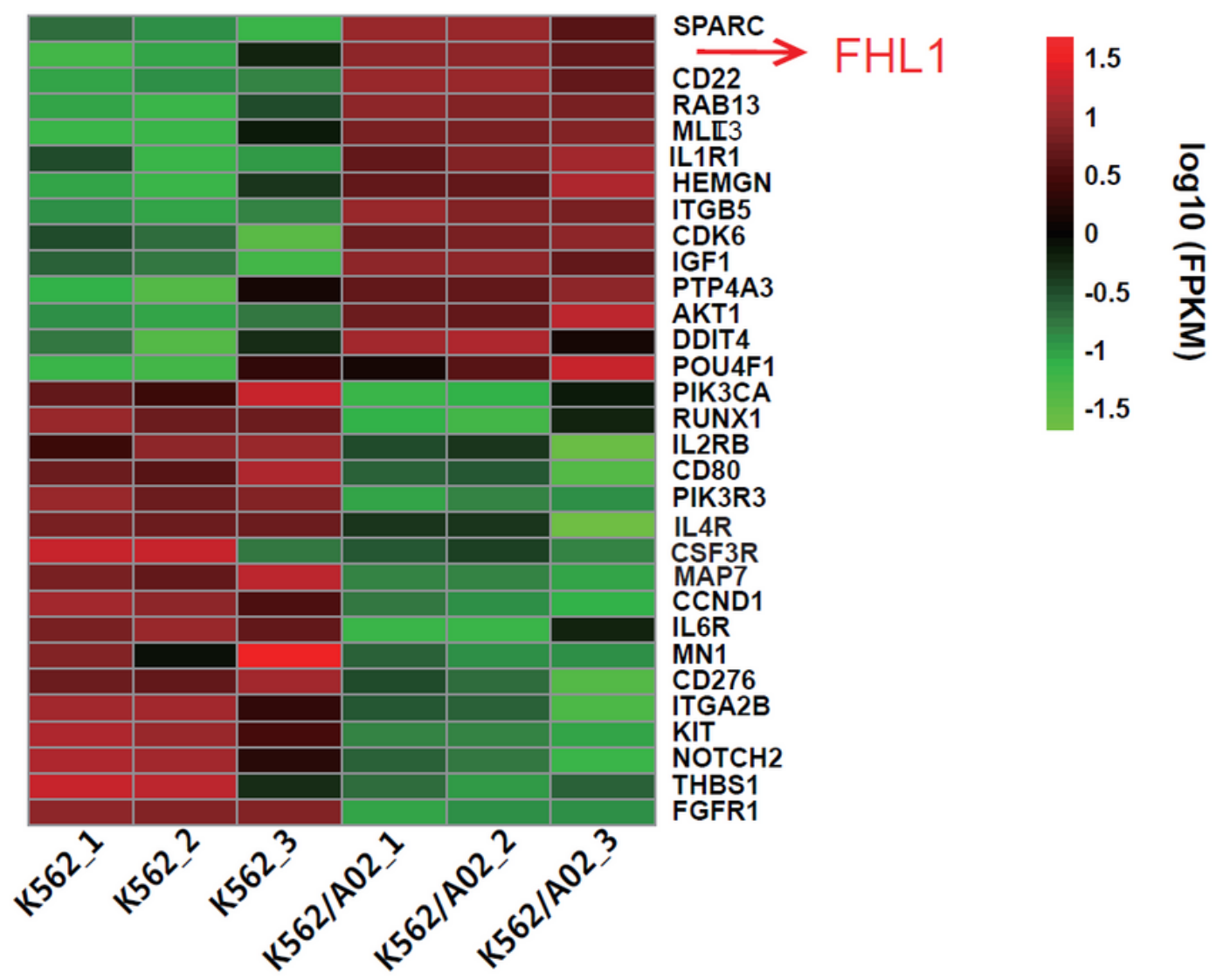

RUNXA

CD80

PIK3R3

IL4R

CSF3R

CCND1

IL6R

MN1

CD276

ITGA2B

KIT

NOTCH2

THBS1

FGFR1

b
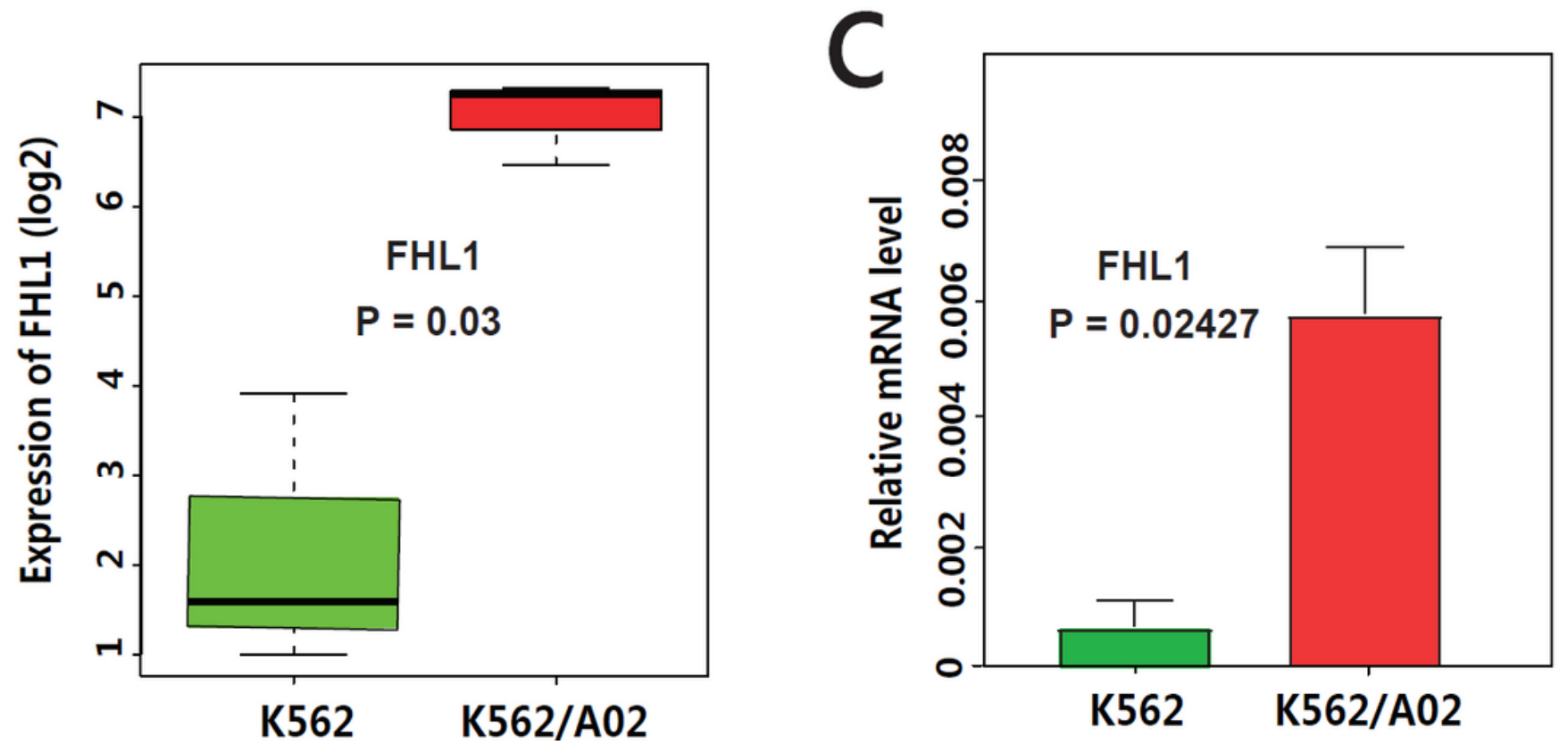

Figure 1

Differential expression between K562/A02 $(n=3)$ and K562 $(n=3)$ AML cell-lines. a Heatmap of associated genes. b Comparison of FHL1's expression. c RT-PCR validation of FHL1 expression. 
a

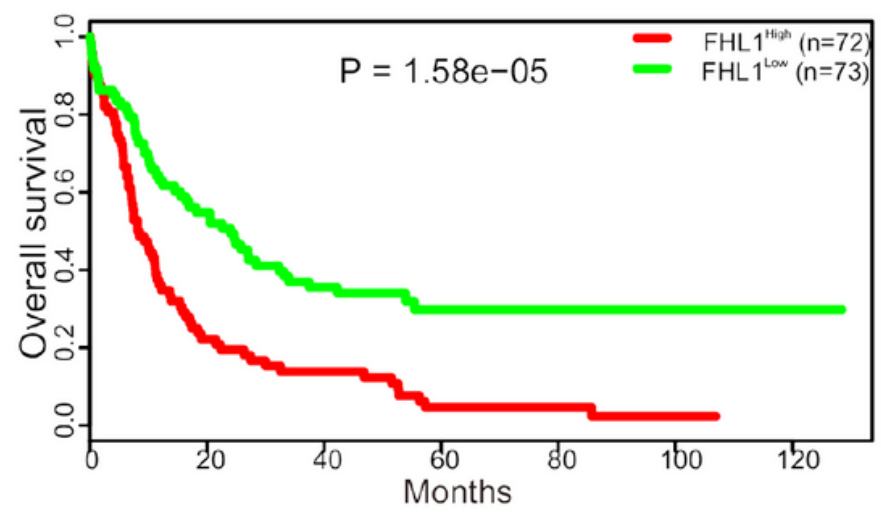

$\mathrm{C}$

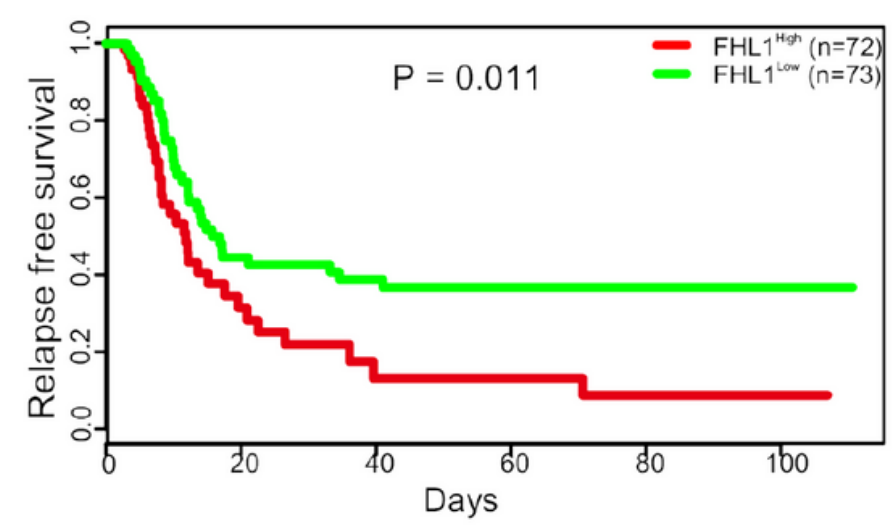

b

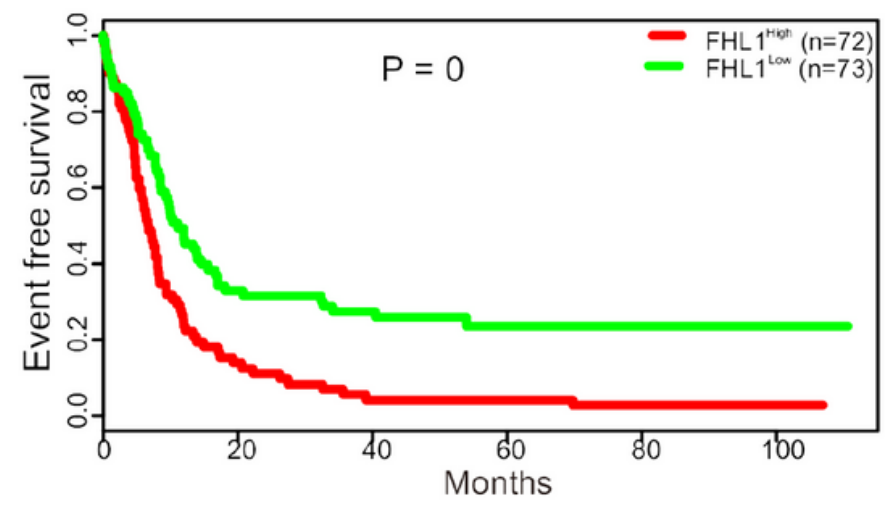

d

\begin{tabular}{|c|c|c|c|c|c|}
\hline \multicolumn{6}{|c|}{ Hazard ratio } \\
\hline Age & $(\mathrm{N}=145)$ & $\begin{array}{c}1.0316 \\
(1.0167-1.047)\end{array}$ & 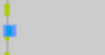 & & $<0.001 \ldots$ \\
\hline FHL1 & $(\mathrm{N}=145)$ & $\begin{array}{c}2.2339 \\
(1.4728-3.388)\end{array}$ & i & $=$ & $<0.001 *$ \\
\hline WBC & $(\mathrm{N}=145)$ & $\begin{array}{c}1.0062 \\
(1.0015-1.011)\end{array}$ & 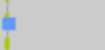 & & $<0.01 *$ \\
\hline NPM1 & $(\mathrm{N}=145)$ & $\begin{array}{c}1.3292 \\
(0.5972-2.958)\end{array}$ & 1 & $\rightarrow$ & 0.486 \\
\hline FLT3 & $(\mathrm{N}=145)$ & $\begin{array}{l}1.304 \\
(0.9904-1.717)\end{array}$ & $\longmapsto$ & & 0.056 \\
\hline CEBPA & $(\mathrm{N}=145)$ & $\begin{array}{c}2.2339 \\
(1.4728-3.388)\end{array}$ & i & $=$ & $<0.001 \cdots$ \\
\hline TP53 & $(\mathrm{N}=145)$ & $\begin{array}{l}28813 \\
(1.5721-5.281)\end{array}$ & $\longmapsto$ & & $<<0.001 \ldots$ \\
\hline $\begin{array}{l}\# C \\
\text { sign }\end{array}$ & $\begin{array}{l}\text { e (Log-R } \\
\cdots 0.001\end{array}$ & $\begin{array}{l}=2 e-10 \\
.01^{\cdot} * 0.05\end{array}$ & 1 & 2 & 10 \\
\hline
\end{tabular}

Figure 2

Prognostic value of FHL1 expression. a Overall survival. b Event-free survival time. c Relapse free survival. d Multivariate Cox regression analysis of FHL1 after taking into account Age, WBC and mutations of NPM1, FLT3, Biallelic CEBPA, TP53 in the 145 IP-AML patients.

a

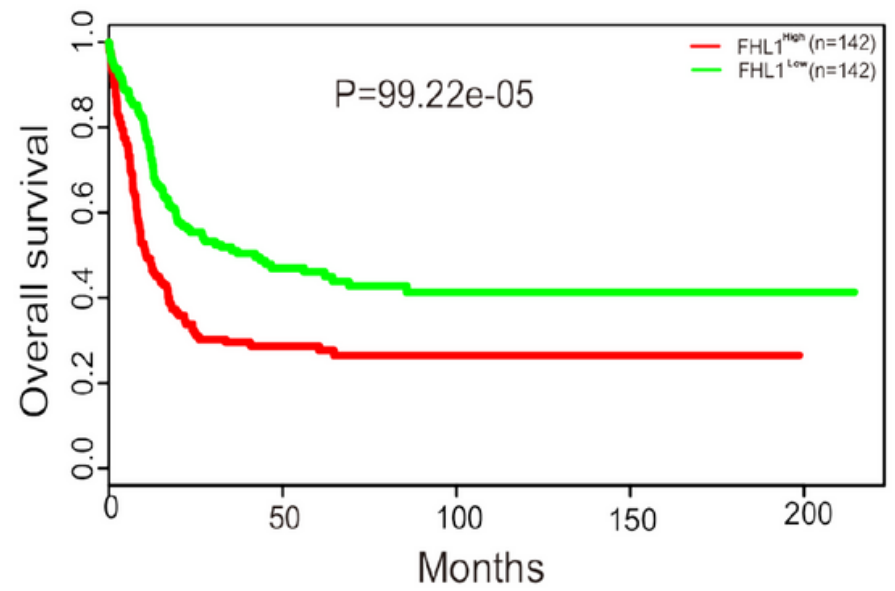

b

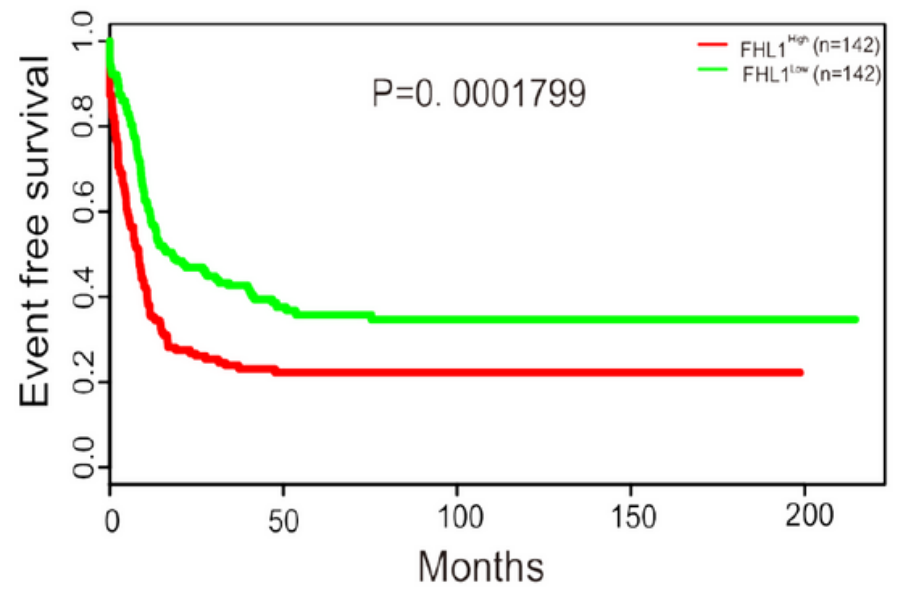

Figure 3 
Prognostic value of FHL1 expression. a Overall survival. b Event-free survival time in the 284 IP-AML patients.

a

b
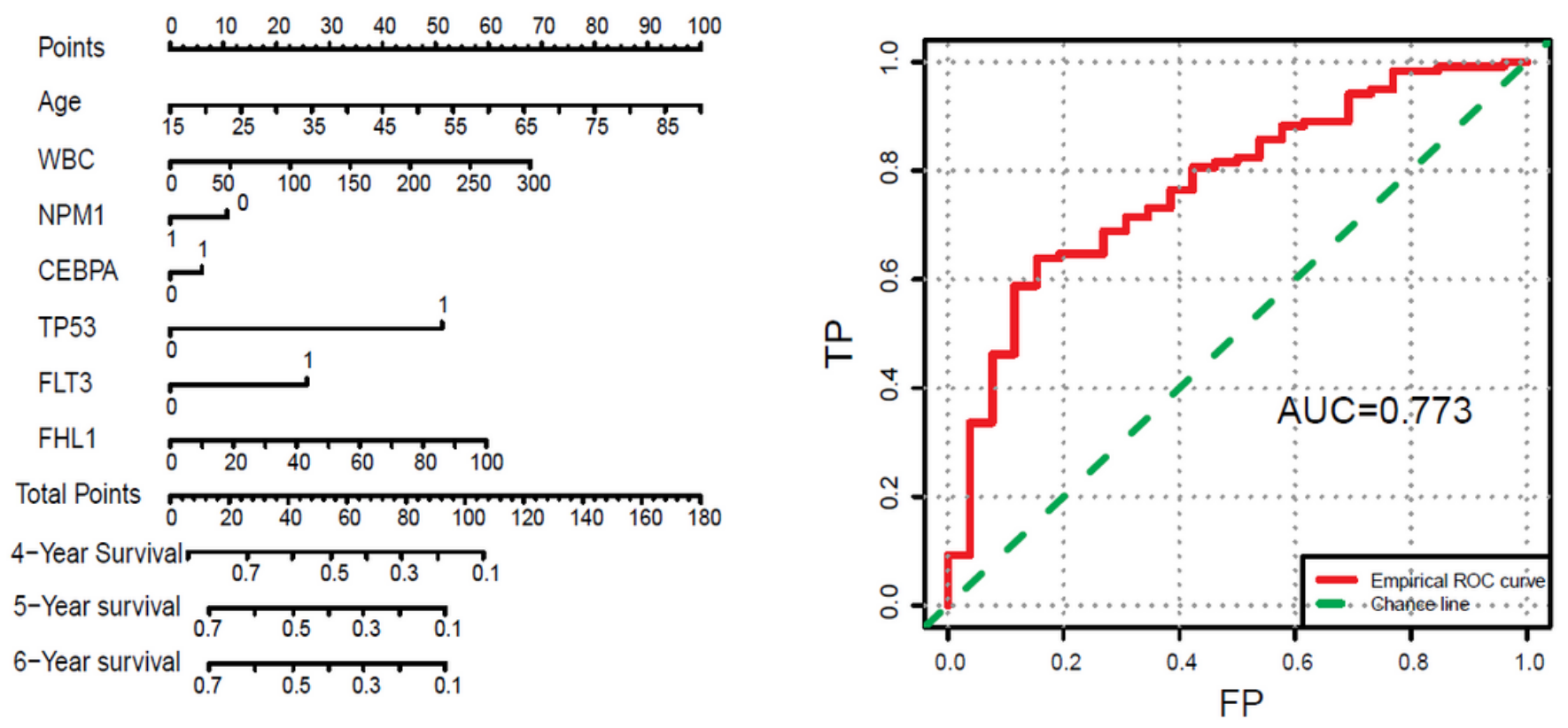

Figure 4

The performance of prognostic model in predicting OS. a Nomogram construction. b ROC curves for OS. 
a

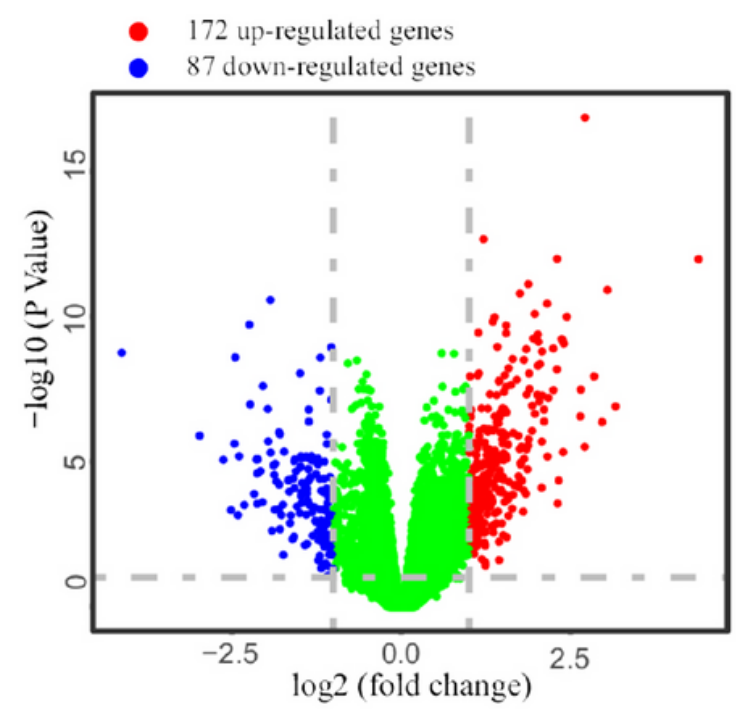

b

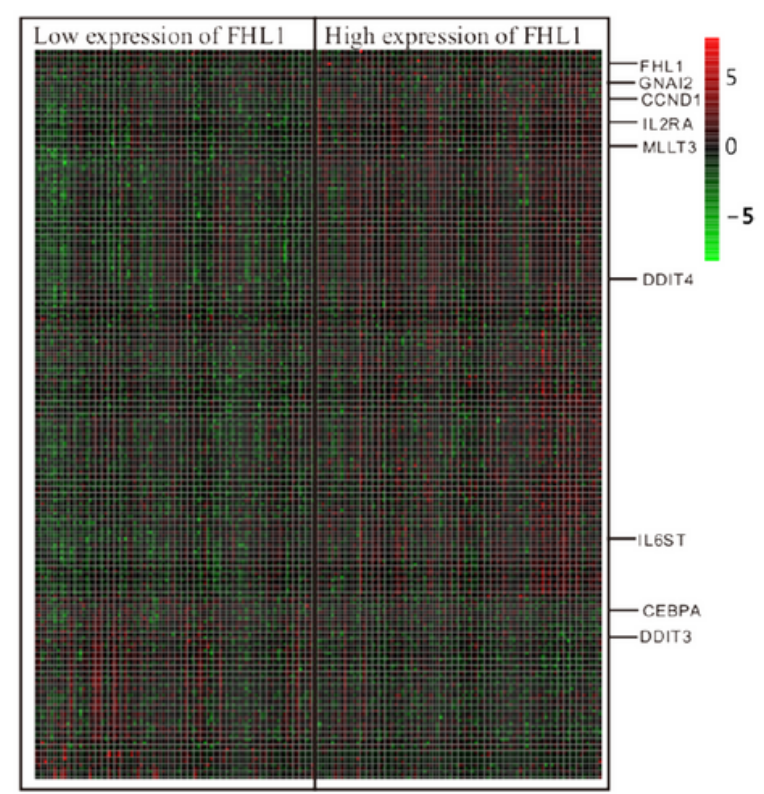

C Hematopoietic cell lineage Cell adhesion molecules (CAMs) Jak-STAT signaling pathway Inflammatory bowel dișease (IBD) Staphylococeus aureus infection Calcium signaling pathway

Pancreatic cancer

Cholinergic synapse

Salivary secretion

Glutathíone metabolism

Ovarian steroidogenesis

insulin signaling pathway

Fc epsilon RI signaling pathway

Inositol phosphate metabolism.

Pathways in cancer

$\mathrm{B}$ cell receptor signaling pathway transmembrane receptor protein tyrosine kinase signaling pathway Jak-STAT signaling pathway $\mathrm{PI} 3 \mathrm{~K}$-Akt signaling pathway $\mathrm{T}$ cell receptor signaling pathway MAPK signaling pathway VEGF signaling pathway Pathways in cancer Platelet activation

cellular response to ketone Transcriptional misregulation in cancer

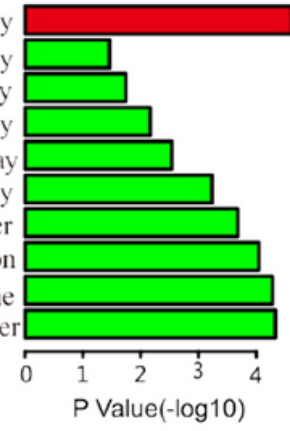

\section{Figure 5}

Genome-wide gene expression profile and cell signaling pathways associated with FHL1 expression. a Volcano plot of differential gene expression in TCGA data. b Expression heat map of associated genes in two group in TCGA data. c Functional enrichment of regulated genes based on gene ontology (GO) annotations in K562/AO2 cell lines and K562 cell lines dataset. d Cell signaling pathways in TCGA data.

\section{Supplementary Files}

This is a list of supplementary files associated with this preprint. Click to download.

- Supplementarytable1.doc 